\title{
Interaction between repeated restraint stress and concomitant midazolam administration on sweet food ingestion in rats
}

P.P. Silveira ${ }^{1}$, M.H. Xavier ${ }^{1}$, F.H. Souza ${ }^{2}$, L.P. Manoli ${ }^{1}$, R.M. Rosat' ${ }^{2}$ M.B.C. Ferreira ${ }^{3}$ and C. Dalmaz ${ }^{1}$
Departamentos de ${ }^{1}$ Bioquímica, ${ }^{2}$ Fisiologia and

${ }^{3}$ Farmacologia, Instituto de Ciências Básicas da Saúde, Universidade Federal do Rio Grande do Sul,

Porto Alegre, RS, Brasil

\section{Correspondence \\ P.P. Silveira \\ Departamento de Bioquímica \\ Laboratório 32, ICBS, UFRGS \\ Ramiro Barcelos, 2600 (Anexo) \\ 90035-003 Porto Alegre, RS \\ Brasil \\ Fax: +55-51-316-5540}

Research supported by PRO NEX (No. 41.96.0904.00).

Received May 31, 1999

Accepted July 13, 2000

\section{Abstract}

Emotional changes can influence feeding behavior. Previous studies have shown that chronically stressed animals present increased ingestion of sweet food, an effect reversed by a single dose of diazepam administered before testing the animals. The aim of the present study was to evaluate the response of animals chronically treated with midazolam and/or submitted to repeated restraint stress upon the ingestion of sweet food. Male adult Wistar rats were divided into two groups: controls and exposed to restraint $1 \mathrm{~h} /$ day, 5 days/week for 40 days. Both groups were subdivided into two other groups treated or not with midazolam $(0.06 \mathrm{mg} / \mathrm{ml}$ in their drinking water during the 40 day treatment). The animals were placed in a lighted area in the presence of 10 pellets of sweet food (Froot loops ${ }^{\circledR}$ ). The number of ingested pellets was measured during a period of $3 \mathrm{~min}$, in the presence or absence of fasting. The group chronically treated with midazolam alone presented increased ingestion when compared to control animals (control group: $2.0 \pm 0.44$ pellets and midazolam group: $3.60 \pm$ 0.57 pellets). The group submitted to restraint stress presented an increased ingestion compared to controls (control group: $2.0 \pm 0.44$ pellets and stressed group: $4.18 \pm 0.58$ pellets). Chronically administered midazolam reduced the ingestion in stressed animals (stressed/ water group: $4.18 \pm 0.58$ pellets; stressed/midazolam group: $3.2 \pm 0.49$ pellets). Thus, repeated stress increases appetite for sweet food independently of hunger and chronic administration of midazolam can decrease this behavioral effect.

\section{Introduction}

Emotional alterations, as well as pharmacological treatments, can influence feeding behavior. For example, food intake is increased by acute administration of diazepam $(1,2)$. Exposure to stressors causes an array of biochemical, physiological and behavior-
Key words

- Feeding behavior

- Chronic stress

- Benzodiazepines

- Midazolam

- Sweet taste

- Rats al changes, and it has been described that chronic exposure to stressors of a certain severity decreases food intake and body weight in the rat $(3,4)$. In addition, increased intake of sucrose has been reported in rats submitted to an inescapable shock session $(3,5)$. Other studies showed that painful tail stimulation produced food craving during 
the training session although the intake was reduced to half in the post-training period $(4,6)$. We have previously observed that chronically stressed animals show increased ingestion of sweet food (7). Studies on humans have provided further evidence of overeating induced by emotional experiences (8).

On the other hand, benzodiazepine agonists, including midazolam, have been claimed to reduce autonomic and hormonal responses to both physiological and psychological stress (9). Acute benzodiazepine administration can also reverse the behavioral effects of stress, such as stress-induced analgesia (10). The effect of chronic stress on appetite for sweet food was reversed by a single dose of diazepam given $60 \mathrm{~min}$ before the test session (7).

Increasing attention has been paid to the chronic use of benzodiazepines. Although these compounds have been reported to increase food intake $(1,2)$, the effect of chronic administration of benzodiazepines has not been well characterized. Here we studied a) the feeding behavior (sweet food intake) of rats submitted to chronic midazolam treatment and b) the effect of this treatment on ingestion after repeated restraint stress.

\section{Material and Methods}

\section{Subjects}

Forty-two experimentally naive adult male Wistar rats (50-70 days old; 150-240 g of weight) were used. The animals were housed in groups of 4-5 in home cages made of Plexiglas $(65 \times 25 \times 15 \mathrm{~cm})$ with the floor covered with sawdust. They were maintained in a controlled environment (lights on between 7:00 $\mathrm{h}$ and 19:00 h, temperature of 22 $\pm 2^{\circ} \mathrm{C}$ ) for at least one week before and throughout the experimental period. Rats had free access to food (standard lab rat chow) and water, except during the period when the behavioral tasks were applied. The immobilization procedure was carried out between 11:00 and 13:00 $\mathrm{h}$ and the behavioral tasks were performed between 15:00 and 16:00 h.

\section{Stress model}

Restraint was applied by placing the animal inside a $25 \times 7-\mathrm{cm}$ plastic tube, and fixing the tube with adhesive tape on the outside, so that the animal was unable to move. There was a $1 \mathrm{~cm}$ hole at the far end for breathing. The animals were submitted to this procedure $1 \mathrm{~h} /$ day, 5 days a week for 40 days and then habituated to the behavioral apparatus and submitted to the task. Stress was maintained throughout this period, which lasted 7 days (see below). Control animals were manipulated but not submitted to restraint. Liquid and lab chow ingestion was measured during the treatment.

\section{Behavioral task}

The animals were placed in a lighted rectangular box $(40 \times 15 \times 20 \mathrm{~cm})$ with floor and side walls made of wood and a glass ceiling. Ten Froot loops (Kellogg's ${ }^{\circledR}$ pellets of wheat and corn starch and sucrose) were placed at one end of the box. Each animal was submitted to 5 days of habituation trials lasting 3 min each, in order to become familiarized with this food. After being habituated, the animal was exposed for $3 \mathrm{~min}$ to 2 test sessions, when the number of ingested pellets was measured. The two tests were performed with the animals submitted to food restriction $(24 \mathrm{~h}$ before the test session the lab chow offered was reduced to about $90 \%$ of the normal amount) or with the animals fed ad libitum. A protocol was established so that when the animal ate part of each Froot loop (e.g., 1/3 or 1/4), this fraction was considered.

\section{Pharmacological treatment}

Midazolam $(0.06 \mathrm{mg} / \mathrm{ml})$ was adminis- 
tered in drinking water as the sole source of water for 40 days to animals submitted or not to restraint stress. The control group received plain water. During the treatment, liquid and food ingestion was measured.

\section{Statistical analysis}

Data are reported as mean \pm SEM and were analyzed by two-way ANOVA, followed by the Duncan test, or by repeated measures ANOVA.

\section{Results}

Food and liquid ingestion was evaluated before each daily stress session. Liquid intake was measured as the difference between the amount of water placed in the drinking bottle and the remaining amount, and is expressed as the mean intake of liquid per rat per $24 \mathrm{~h}$. Results are shown in Table 1. Repeated measures ANOVA showed an effect of chronic midazolam treatment $(\mathrm{F}(1,71)=11.05 ; \mathrm{P}<0.01)$, with midazolamtreated animals presenting higher ingestion when compared to the other groups. There was no effect of time $(\mathrm{F}(3,213)=0.49$; $\mathrm{P}>0.05)$ or of chronic stress $(\mathrm{F}(1,71)=0.12$; $\mathrm{P}>0.05)$. The interaction between midazolam and stress treatment was not significant $(\mathrm{P}=0.058)$. The mean midazolam dose administered to the animals was $8.91 \mathrm{mg} \mathrm{kg}^{-1}$ $24 \mathrm{~h}^{-1}$. Food intake was measured by weighing the amount of food placed in the feeders and that remaining in them, and is expressed as grams of food consumed per animal within $24 \mathrm{~h}$. This was averaged across 10-day blocks, as shown in Table 2. Repeated measures ANOVA showed no effect of chronic stress $(\mathrm{F}(1,51)=2.77 ; \mathrm{P}>0.1)$ or of chronic midazolam treatment $(\mathrm{F}(1,51)=2.54 ; \mathrm{P}>0.1)$, but there was an interaction between midazolam and stress treatment $(\mathrm{F}(1,51)=4.14 ; \mathrm{P}<0.05)$; there was an effect of time $(\mathrm{F}(3,153)=4.40$; $\mathrm{P}<0.01)$, and an interaction between stress and time $(\mathrm{F}(1,12)=3.95 ; \mathrm{P}<0.05)$. Body weight was measured at the beginning and at the end of treatment, and is shown in Table 3. Repeated measures ANOVA showed an effect of time $(\mathrm{F}(1,34)=635.44 ; \mathrm{P}<0.001)$, since all groups gained weight. There was no effect of chronic midazolam treatment $(\mathrm{F}(1,34)=0.0 ; \mathrm{P}>0.05)$, or of exposure to chronic stress $(\mathrm{F}(1,34)=3.25 ; \mathrm{P}=0.08)$.

However, there was a significant stress $\mathrm{x}$

Table 1 - Mean \pm SEM intake of water during chronic treatments.

Data are reported as $\mathrm{ml}$ of water ingested per animal per $24 \mathrm{~h} . \mathrm{N}=9-12$ animals/group. Mdz, Midazolam.

\begin{tabular}{lcccc}
\hline Group & Control & Stress & Mdz & Mdz + Stress \\
\hline Days 1-10 & $50.1 \pm 3.1$ & $44.9 \pm 2.8$ & $59.9 \pm 3.4$ & $55.2 \pm 3.2$ \\
Days 11-20 & $49.4 \pm 3.1$ & $45.8 \pm 2.8$ & $50.9 \pm 3.3$ & $60.8 \pm 3.5$ \\
Days 21-30 & $51.0 \pm 2.7$ & $43.5 \pm 1.7$ & $49.8 \pm 1.9$ & $58.5 \pm 3.6$ \\
Days 31-40 & $50.9 \pm 4.7$ & $47.4 \pm 3.5$ & $52.4 \pm 3.9$ & $55.9 \pm 4.6$
\end{tabular}

Table 2 - Food ingestion during chronic restraint stress, measured throughout the 50 days of treatment.

Data are arranged in 10-day blocks and expressed as mean \pm SEM ingestion of food per animal. There was no difference between groups as determined by repeated measures ANOVA ( $P>0.05)$. Mdz, Midazolam.

\begin{tabular}{lcccc}
\hline Days of treatment & $1-10$ & $11-20$ & $21-30$ & $31-40$ \\
\hline Control/water & $26.2 \pm 0.62$ & $24.4 \pm 1.02$ & $23.4 \pm 1.02$ & $25.4 \pm 1.17$ \\
Stressed/water & $21.4 \pm 0.99$ & $23.0 \pm 0.83$ & $21.3 \pm 1.12$ & $21.2 \pm 0.79$ \\
Control/Mdz & $27.3 \pm 1.86$ & $23.8 \pm 0.72$ & $21.7 \pm 1.21$ & $25.2 \pm 1.35$ \\
Stressed/M dz & $22.4 \pm 0.82$ & $24.0 \pm 0.57$ & $22.4 \pm 0.79$ & $29.5 \pm 3.70$
\end{tabular}

Table 3 - Body weight (g) during chronic restraint stress, measured throughout the 50 days of treatment.

Data are reported as mean \pm SEM. There was no difference between groups, but there was a significant group $x$ time interaction as determined by repeated measures ANOVA $(\mathrm{P}<0.001)$. Mdz, Midazolam.

\begin{tabular}{lcc}
\hline & Before treatment & After treatment \\
\hline Control/water & $225.1 \pm 11.17$ & $335.0 \pm 13.03$ \\
Stressed/water & $208.7 \pm 5.70$ & $293.3 \pm 11.49$ \\
Control/Mdz & $211.2 \pm 12.33$ & $324.7 \pm 11.72$ \\
Stressed/Mdz & $214.1 \pm 6.70$ & $310.0 \pm 8.60$
\end{tabular}


time interaction $(\mathrm{F}(1,34)=7.16 ; \mathrm{P}<0.02)$, which means that the stressed rats gained less weight than controls. There was no midazolam $\mathrm{x}$ time interaction $(\mathrm{F}(1,34)=0.86$; $\mathrm{P}>0.05$ ).

Chronic midazolam treatment, as well as repeated restraint stress, induced an increased intake of Froot loops, whether or not the animals ( $\mathrm{N}=9-12$ /group) were submitted to food restriction. In addition, chronic midazolam decreased the effect of restraint, since animals submitted to both stress and midazolam presented a different intake compared to animals submitted to stress alone, although still higher than that of control rats. In the fed state (Figure 1A), two-way ANOVA revealed a significant interaction between chronic midazolam treatment and chronic stress $(\mathrm{F}(1,38)=5.509 ; \mathrm{P}<0.05)$. The chronically stressed group presented a higher ingestion of sweet pellets compared to the control group ( $\mathrm{P}<0.05$, Duncan test), and chronic treatment with midazolam reduced this effect. Under food restriction (Figure 1B), twoway ANOVA revealed a significant effect of

Figure 1 - Number of Froot loops consumed during the test sessions. A, Animals tested in the fed state. B, Animals tested under food restriction. Data are reported as mean \pm SEM. There was a significant interaction between chronic midazolam (Mdz) treatment and chronic stress (two-way ANOVA; $\mathrm{P}<0.05$ ). N = 9-12 animals/group. $* \mathrm{P}<0.05$ compared to control/water group. $+\mathrm{P}<0.05$ compared to stressed/water group.
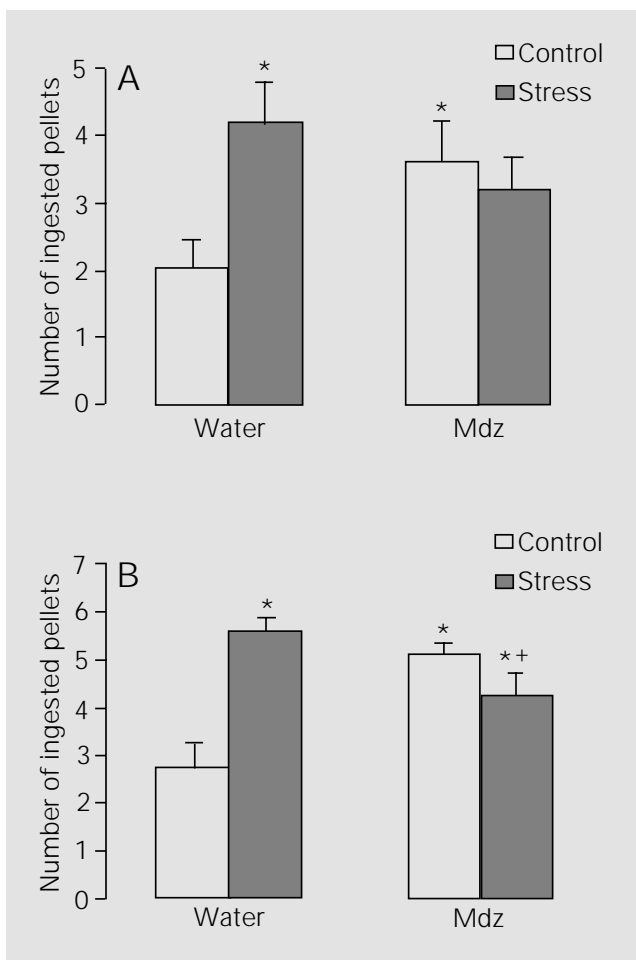

chronic stress $(\mathrm{F}(1,39)=5.12, \mathrm{P}<0.05)$ causing increased intake. There was also a significant interaction between chronic midazolam treatment and chronic stress $(\mathrm{F}(1,39)$ $=19.99 ; \mathrm{P}<0.001)$.

\section{Discussion}

Midazolam (8-chloro-6-(2-fluorophenyl)1-methyl-4H-imidazo [1,5-a] [1,4] benzodiazepine) is a water-soluble benzodiazepine, stable in aqueous solution. The short halflives of midazolam and its active metabolites allow daily dosing without the accumulation of these substances (11).

In agreement with previous observations (7), we report an increased appetite for sweet food in response to repeated stress independently of hunger. In addition, there was an increased ingestion of sweet food in animals chronically treated with midazolam. No alteration of intake of habitual ration was observed in the groups submitted to chronic restraint or chronic midazolam alone. The interaction between stress and midazolam treatment was shown by the increased ingestion of food in the group submitted to both treatments, when compared to the groups receiving one of the treatments only. All groups gained weight and no difference between groups was found during the entire treatment, although there was a significant interaction between stress and time.

Effects of drugs acting as agonists of benzodiazepine receptors, including midazolam, on food intake have been reported. Acutely administered benzodiazepines produce many interesting behavioral effects, among them the stimulation of food, water and salt intake in many different species (1214). In our study, chronic midazolam administration increased water intake, particularly in stressed rats, although there was no significant interaction. The anorectic effects of acutely administered inverse agonists have been described (12). The non-benzodiazepine anxiolytics, zopiclone and CL 218,872, 
also produce significant increases in food intake (15). In contrast, the benzodiazepine agonist zolpidem has no effect on food intake (16), providing pharmacological evidence for a dissociation between hyperphagic and anxiolytic or hypnotic drug effects (12, 15-17). These studies were done using drugs administered in a single dose.

Acute administration of the benzodiazepine receptor agonist midazolam at doses similar to those used in the present study significantly increased food intake $(12,18)$. It was shown that the increase in food consumption observed after midazolam administration was due to increases in the duration of feeding, specifically in the duration of eating bouts, but not in their frequency (18). Midazolam has also been shown to increase consumption of sucrose solutions or of sweet food when administered both systemically or centrally $(12,13)$, and flumazenil blocks this effect (13). These studies evaluated the consumption of sweet food or solutions after acute doses of midazolam. In our study, it is interesting to note that the increase in sweet food consumption was observed after chronic treatment with this drug, although there was no effect on the consumption of regular food.

Other studies $(3,4)$ have reported that chronic exposure to stressors of a certain severity decreases food intake and body weight of rats. However, the type, duration or severity of stress and the different strains and gender of the experimental animals used may modify the responses to stress $(4,19,20)$. Models using more severe stressors have also shown effects on feeding behavior, in which the animals present decreased intake of food, including lab chow, and not only of sweet food. For example, inescapable shock can profoundly affect ingestion and reduce weight gain, with shocked rats gaining significantly less weight than restrained rats (3). When considering chronic mild stress, different effects have also been observed. Some models of chronic variate stress have been proposed as models of depression in animal studies $(20,21)$. In these models, the rats are exposed to different weak stressors for several days, and even the response to rewarding stimuli is diminished, as demonstrated by tests including sucrose consumption, which is reduced, a fact interpreted as anhedonia. This effect is reversed by antidepressants (22). Comparing these different models of stress to ours, we observed that, although mild stressors are used in both models, in the present study repeated restraint stress was applied, which implies a certain degree of predictability, while those models used different stressors $(21,22)$. Therefore, different models of stress can have different effects on feeding behavior.

Increased consumption of carbohydrates following exposure to stress has been observed in humans (23). Patients who become fat when exposed to stress sometimes present the tendency to overeat carbohydrates to make themselves feel better. This effect is believed to be related to the property of carbohydrate consumption, acting via insulin secretion and the "plasma tryptophan ratio" to increase serotonin release, since serotonin release is also involved in functions such as mood control (24).

The central mechanisms involved in stress-induced overeating are very complex. Many agents such as $\alpha$-adrenoceptor agonists, beta-endorphin, dynorphin, neuropeptide Y (NPY) and galanine stimulate food intake (25). The effects of pharmacological and behavioral treatments on the hedonic response to feeding are another important dimension of eating behavior. Studies suggest that mechanisms involving central dopamine, opioid peptides and serotonin systems are necessary for a normal eating response to sweet tasting stimuli independently of hunger perception (25). Several hormones released in response to stress are known to influence feeding. For example, opioids are proposed to play a role in the control of food intake since acute administration of opioids increases food intake 
$(26,27)$ while administration of opioid antagonists decreases food intake, either administered acutely or in a 7-day schedule (26), and their ability to do it appears to be dependent on the palatability of the food $(26,28)$. It appears that excess opioid agonist activity may result in hyperphagia or anorexia (depending on the opiate receptor type) (27).

Corticotropin-releasing hormone (CRH), the main regulator of the hypothalamic-pituitary-adrenal axis, also participates in the regulation of appetite and energy expenditure $(29,30)$. It has been suggested that hypothalamic CRH systems in the paraventricular nucleus exert inhibitory control over NPYinduced food intake (30). CRH administration impairs intake, and administration of CRH antagonists facilitates the intensity of the feeding response to NPY or exposure to stress, such as tail pinch (31) or restraint (32). The anorectic effect of CRH decreased over repeated injections (33), so it is probably not involved in the effects of chronic stress on appetite.

Glucocorticoids may also induce alterations in appetite. Therapeutic doses of glucocorticoids in humans increase energy intake, an effect which may be related to the ability of glucocorticoids to act directly or indirectly on the central regulation of appetite (34). Adrenalectomy induces anorexia in mice, while corticosterone replacement completely blocks this effect (35). Glucocorticoid levels within the physiological range can interfere with the action of leptin, reversing its effects, and these effects are at least partly independent of NPY (36). Nevertheless, other studies in humans (37) and rats (38) found glucocorticoid-induced increases in leptin secretion. Although acute treatment with the synthetic corticosteroid dexamethasone resulted in a stimulatory effect on leptin secretion and expression, chronic dexamethasone treatment did not
(38). The divergence between the appetitestimulating effects of glucocorticoids despite induction of a proposed satiety factor suggests that regulation of appetite is multifactorial, and other neurotransmitter pathways are presumably involved. Endogenous corticosterone has a permissive effect on the carbohydrate-feeding responses elicited by norepinephrine and NPY in the paraventricular nucleus (39). Chronic restraint stress could influence any of the above mechanisms stimulating the appetite for sweet food.

It is important to note that this increased ingestion of food is not observed with peanuts, which is a palatable food. Stressed animals show no different ingestion when compared to controls (data not shown).

The effect of chronic midazolam on the overeating associated with chronic restraint suggests that anxiety related to this effect of stress was an important factor influencing eating behavior. In this experiment, rats chronically treated with oral midazolam did not present tolerance to its effects on ingestive behavior concerning sweet food or on anxiety. On the other hand, it has been proposed that the effect of diazepam on food intake is dependent on the activation of endogenous opioids (40). It is not known if the effect of chronic midazolam on sweet food would also involve stimulation of this system.

These results lead to the conclusion that the severity and duration of exposure to stressors are capable of modifying eating behavior. Chronic moderate stress like restraint does not alter normal food consumption but leads to changes in specific appetites expressed in this study by greater sweet food ingestion. This difference may be the result of an alteration in the latencies to eat or in the velocity. This alteration was probably due to a higher level of anxiety, since it was reversed by acute diazepam administration (7) or by chronic midazolam. 


\section{References}

1. Britton DR, Britton $K T$, Dalton $D \&$ Vale W (1981). Effects of naloxone on anti-conflict and hyperphagic actions of diazepam. Life Sciences, 29: 1297-1302.

2. Cooper SJ (1983). Benzodiazepine-opiate antagonist interactions in relation to anxiety and appetite. Trends in Pharmacological Sciences, 4: 456-458.

3. Dess NK, Raizer J , Chapman CD \& Garcia J (1988). Stressors in the learned helplessness paradigm: effects on body weight and conditioned taste aversion in rats. Physiology and Behavior, 44: 483490.

4. Marti O, Marti J \& Armario A (1994). Effect of chronic stress on food intake in rats: Influence of stressor intensity and duration of daily exposure. Physiology and Behavior, 55: 747-753.

5. Dess NK (1992). Divergent responses to saccharin vs sucrose availability after stress in rats. Physiology and Behavior, 52: 105-125.

6. Rowland N \& Antelman SM (1976). Stress-induced hyperphagia and obesity in rats: a possible model for understanding human obesity. Science, 191: 310312.

7. Ely DR, Dapper V, Marasca J, Corrêa J B, Gamaro GD, Xavier MH, Michalowski MB, Catelli D, Rosat R, Ferreira MBC \& Dalmaz C (1997). Effect of restraint stress on feeding behavior of rats. Physiology and Behavior, 61: 395-398.

8. Yates A (1992). Biological considerations in the aetiology of eating. Pediatric Annals, 21: 739-744.

9. Nilsson A (1990). Autonomic and hormonal responses after the use of midazolam and flumazenil. Acta Anaesthesiologica Scandinavica, 34 (Suppl 92): 51-54.

10. Willer J C \& Ernst M (1986). Somatovegetative changes in stress-induced analgesia in man: an electrophysiological and pharmacological study. Annals of the New York Academy of Sciences, 467: 256-272.

11. Hardman J G, Gilman AG \& Limbird LE (Editors) (1996). Goodman \& Gilman's The Pharmacological Basis of Therapeutics. 9th edn. McGraw-Hill, New York.

12. Yerbury RE \& Cooper SJ (1987). The benzodiazepine partial agonists, Ro 16-6028 and Ro 17-1812, increase palatable food consumption in nondeprived rats. Pharmacology, Biochemistry and Behavior, 28: 427-431.

13. Higgs S \& Cooper SJ (1996). Increased food intake following injection of the ben- zodiazepine receptor agonist midazolam into the IVth ventricle. Pharmacology, Biochemistry and Behavior, 55: 81-86.

14. Lobarinas E \& Falk J L (1998). Scheduleinduced polydipsic consumption of hypertonic $\mathrm{NaCl}$ solutions: Effects of chlordiazepoxide. Physiology and Behavior, 63: 419-423.

15. Cooper SJ \& Moores WR (1985). Benzodiazepine-induced hyperphagia in the nondeprived rat: comparisons with $\mathrm{CL}$ 218,872, zopiclone, tracazolate and phenobarbital. Pharmacology, Biochemistry and Behavior, 23: 169-172.

16. Yerbury RE \& Cooper SJ (1989). Novel benzodiazepine receptor ligands: palatable food intake following zolpidem, CGS 17867A, or Ro23-0364, in the rat. Pharmacology, Biochemistry and Behavior, 33: 303-307.

17. Cooper SJ , Yerbury RE, Neill J C \& Desa A (1987). Partial agonists acting at benzodiazepine receptors can be differentiated in tests of ingestional behavior. Physiology and Behavior, 41: 247-255.

18. Cooper SJ \& Yerbury RE (1986). Midazolam-induced hyperphagia and FG 7142induced anorexia: behavioural characteristics in the rat. Pharmacology, Biochemistry and Behavior, 25: 99-106.

19. Paré WP \& Redei E (1993). Sex differences and stress response of WKY rats. Physiology and Behavior, 54: 1179-1185.

20. Pucilowski O, Overstreet $\mathrm{DH}$, Rezvani $A H$ \& J anowsky DS (1993). Chronic mild stress-induced anhedonia: greater effect in a genetic rat model of depression. Physiology and Behavior, 54: 1215-1220.

21. Katz RJ (1981). Animal models and human depressive disorders. Neuroscience and Biobehavioral Reviews, 5: 231-246.

22. Willner $P$, Towell A, Sampson D, Sophokleous S \& Muscat R (1987). Reduction of sucrose preference by chronic unpredictable mild stress, and its restoration by a tricyclic antidepressant. Psychopharmacology, 93: 358-364.

23. Levine MD \& Marcus MD (1997). Eating behavior following stress in women with and without bulimic symptoms. Annals of Behavioral Medicine, 19: 132-138.

24. Wurtman RJ \& Wurtman JJ (1995). Brain serotonin, carbohydrate-craving, obesity and depression. Obesity Research, 4 (Suppl): 477S-480S.

25. Blundell J (1991). Pharmacological approaches to appetite suppression. Trends in Pharmacological Sciences, 12: 147-157.
26. McLaughlin CL \& Baile CA (1983). Nalmefene decreases meal size, food and water intake and weight gain in Zucker rats. Pharmacology, Biochemistry and Behavior, 19: 235-240.

27. Yim GK \& Lowy MT (1984). Opioids, feeding, and anorexias. Federation Proceedings, 43: 2893-2897.

28. Giraudo SQ, Grace MK, Welch CC Billington CJ \& Levine AS (1993). Naloxone's anorectic effect is dependent upon the relative palatability of food. Pharmacology, Biochemistry and Behavior, 46: 917-921.

29. Kalra SP, Dube MG, Pu S, Xu B, Horvath TL \& Kalra PS (1999). Interacting appetiteregulating pathways in the hypothalamic regulation of body weight. Endocrine Reviews, 20: 68-100.

30. Heinrichs SC, Menzaghi F, Pich EM, Hauger RL \& Koob GF (1993). Corticotropin-releasing factor in the paraventricular nucleus modulates feeding induced by neuropeptide Y. Brain Research, 611: 1824.

31. Heinrichs SC, Cole BJ , Pich EM, Menzaghi F, Koob GF \& Hauger RL (1992). Endogenous corticotropin-releasing factor modulates feeding induced by neuropeptide $Y$ or a tail-pinch stressor. Peptides, 13: 879884.

32. Heinrichs SC \& Koob GF (1992). Corticotropin-releasing factor modulates dietary preference in nutritionally and physically stressed rats. Psychopharmacology, 109: 177-184.

33. Krahn DD, Gosnell BA \& Majchrzak MJ (1990). The anorectic effects of CRH and restraint stress decrease with repeated exposures. Biological Psychiatry, 27: 1094-1102.

34. Tataranni PA, Larson DE, Snitker S, Young J B, Flatt J P \& Ravussin E (1996). Effects of glucocorticoids on energy metabolism and food intake in humans. American J ournal of Physiology, 271 (Part 1): E317E325.

35. J acobson L (1999). Glucocorticoid replacement, but not corticotropin-releasing hormone deficiency, prevents adrenalectomy-induced anorexia in mice. Endocrinology, 140: 310-317.

36. Solano J M \& J acobson L (1999). Glucocorticoids reverse leptin effects on food intake and body fat in mice without increasing NPY mRNA. American J ournal of Physiology, 277 (Part 1): E708-E716.

37. Miell J P, Englaro P \& Blum WF (1996). 
Dexamethasone induces an acute and sustained rise in circulating leptin levels in normal human subjects. Hormone and Metabolic Research, 28: 704-707.

38. Tan J T, Patel BK, Kaplan LM, Koenig J I \& Hooi SC (1998). Regulation of leptin expression and secretion by corticosteroids and insulin. Implications for body weight Endocrine, 8: 85-92.

39. Tempel DL \& Leibowitz SF (1993). Glucocorticoid receptors in PVN: interactions with NE, NPY, and Gal in relation to feeding. American J ournal of Physiology, 265 (5 Pt 1): E794-E800.
40. Gonzalez Y, Fernandez-Tome MP, Sanchez-Franco F \& del Rio J (1984). Antagonism of diazepam-induced feeding in rats by antisera to opioid peptides. Life Sciences, 35: 1423-1429. 